\title{
Small-scale power spectrum of cold dark matter
}

\section{Citation}

Loeb, Abraham, and Matias Zaldarriaga. 2005. "Small-Scale Power Spectrum of Cold Dark Matter." Physical Review D 71 (10). https://doi.org/10.1103/physrevd.71.103520.

\section{Permanent link}

http://nrs.harvard.edu/urn-3:HUL.InstRepos:41417261

\section{Terms of Use}

This article was downloaded from Harvard University's DASH repository, and is made available under the terms and conditions applicable to Other Posted Material, as set forth at http:// nrs.harvard.edu/urn-3:HUL.InstRepos:dash.current.terms-of-use\#LAA

\section{Share Your Story}

The Harvard community has made this article openly available.

Please share how this access benefits you. Submit a story.

Accessibility 


\title{
The Small-Scale Power Spectrum of Cold Dark Matter
}

\author{
Abraham Loeb \& Matias Zaldarriaga ${ }^{\star}, \dagger$

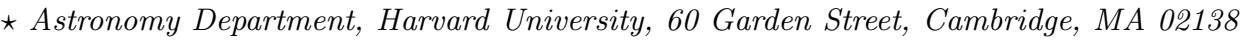 \\ $\dagger$ Physics Department, Harvard University, 17 Oxford Street, Cambridge MA 02138
}

(February 2, 2008)

\begin{abstract}
One of the best motivated hypotheses in cosmology states that most of the matter in the universe is in the form of weakly-interacting massive particles that decoupled early in the history of the universe and cooled adiabatically to an extremely low temperature. Nevertheless, the finite temperature and horizon scales at which these particles decoupled imprint generic signatures on their small scale density fluctuations. We show that the previously recognized cut-off in the fluctuation power-spectrum due to free-streaming of particles at the thermal speed of decoupling, is supplemented by acoustic oscillations owing to the initial coupling between the cold dark matter (CDM) and the radiation field. The power-spectrum oscillations appear on the scale of the horizon at kinematic decoupling which corresponds to a mass scale of $\sim 10^{-4}\left(T_{\mathrm{d}} / 10 \mathrm{MeV}\right)^{-3} M_{\odot}$ for a CDM decoupling temperature $T_{\mathrm{d}}$. The suppression of the power-spectrum on smaller scales by the acoustic oscillations is physically independent from the free-streaming effect, although the two cut-off scales are coincidentally comparable for $T_{\mathrm{d}} \sim 10 \mathrm{MeV}$ and a particle mass of $M \sim 100 \mathrm{GeV}$. The initial conditions for recent numerical simulations of the earliest and smallest objects to have formed in the universe, need to be modified accordingly. The smallest dark matter clumps may be detectable through $\gamma$-ray production from particle annihilation, through fluctuations in the event rate of direct detection experiments, or through their tidal gravitational effect on wide orbits of objects near the outer edge of the solar system.
\end{abstract}

PACS numbers: 95.35.+d, 14.80.Ly, 98.35.Ce, 98.80.Cq

Introduction. A broad range of observational data involving the dynamics of galaxies, the growth of largescale structure, and the dynamics and nucleosynthesis of the universe as a whole, indicate the existence of dark matter with a mean cosmic mass density that is $\sim 5$ times larger than the density of the baryonic matter $[1,2]$. The data is consistent with a dark matter composed of weakly-interacting, massive particles, that decoupled early and adiabatically cooled to an extremely low temperature by the present time [1]. The Cold Dark Matter (CDM) has not been observed directly as of yet, although laboratory searches for particles from the dark halo of our own Milky-Way galaxy, have been able to restrict the allowed parameter space for these particles. Since an alternative more-radical interpretation of the dark matter phenomenology involves a modification of gravity [3], it is of prime importance to find direct fingerprints of the CDM particles. One such fingerprint involves the small-scale structure in the universe [4], on which we focus in this paper.

Perhaps the most popular candidate for the CDM particle is a Weakly Interacting Massive Particle (WIMP). The lightest supersymmetric particle (LSP) could be a WIMP (for a review see [1]). The CDM particle mass depends on free parameters in the particle physics model but typical values cover a range around $M \sim 100 \mathrm{GeV}$ (up to values close to a TeV). In many cases the LSP hypothesis will be tested at the Large Hadron Collider (e.g. [5]) or in direct detection experiments (e.g. [6]).

The properties of the CDM particles affect their re- sponse to the small-scale primordial inhomogeneities produced during cosmic inflation. The particle cross-section for scattering off standard model fermions sets the epoch of their thermal and kinematic decoupling from the cosmic plasma (which is significantly later than the time when their abundance freezes-out at a temperature $T \sim$ $M)$. Thermal decoupling is defined as the time when the temperature of the CDM stops following that of the cosmic plasma while kinematic decoupling is defined as the time when the bulk motion of the two species start to differ. For CDM the epochs of thermal and kinetic decoupling coincide. They occur when the time it takes for collisions to change the momentum of the CDM particles equals the Hubble time. The particle mass determines the thermal spread in the speeds of CDM particles, which tends to smooth-out fluctuations on very small scales due to the free-streaming of particles after kinematic decoupling $[4,7]$. Viscosity has a similar effect before the CDM fluid decouples from the cosmic radiation fluid [8]. An important effect that has been previously ignored involves the memory the CDM fluid has of the acoustic oscillations of the cosmic radiation fluid out of which it decoupled. Here we consider the imprint of these acoustic oscillations on the small-scale power spectrum of density fluctuations in the universe. Other imprints of acoustic oscillations on much larger scales were identified recently in maps of the Cosmic Microwave Background (CMB) [2], and the distribution of nearby galaxies [9].

Throughout this paper, we adopt the standard set of cosmological parameters [2] for a universe dominated by 
cold dark matter and a cosmological constant $(\Lambda-\mathrm{CDM})$.

Formalism Kinematic decoupling of CDM occurs during the radiation-dominated era. For example, if the CDM is made of neutralinos with a particle mass of $\sim 100 \mathrm{GeV}$, then kinematic decoupling occurs at a cosmic temperature of $T_{\mathrm{d}} \sim 10 \mathrm{MeV}[8,10]$. As long as $T_{d} \ll 100 \mathrm{MeV}$, we may ignore the imprint of the QCD phase transition on the CDM power spectrum [11]. Over a short period of time during this transition, the sound speed of the plasma vanishes, resulting in a significant growth for perturbations with periods shorter than the length of time over which the sound speed vanishes. The transition occurs when the temperature of the cosmic plasma is $\sim 100-200 \mathrm{MeV}$ and lasts for a small fraction of the Hubble time. As a result, the induced modifications are on scales smaller than those we are considering here and the imprint of the QCD phase transition is washed-out by the effects we calculate.

At early times the contribution of the dark matter to the energy density is negligible. Only at relatively late times when the cosmic temperature drops to values as low as $\sim 1 \mathrm{eV}$, matter and radiation have comparable energy densities. As a result, the dynamics of the plasma at earlier times is virtually unaffected by the presence of the dark matter particles. In this limit, the dynamics of the radiation determines the gravitational potential and the dark matter just responds to that potential. We will use this simplification to obtain analytic estimates for the behavior of the dark matter transfer function.

The primordial inflationary fluctuations lead to acoustic modes in the radiation fluid during this era. The interaction rate of the particles in the plasma is so high that we can consider the plasma as a perfect fluid down to a comoving scale,

$$
\lambda_{f} \sim \eta_{d} / \sqrt{N} \quad ; \quad N \sim n \sigma t_{d},
$$

where $\eta_{d}=\int_{0}^{t_{d}} d t / a(t)$ is the conformal time (i.e. the comoving size of the horizon) at the time of CDM decoupling, $t_{d} ; \sigma$ is the scattering cross section and $n$ is the relevant particle density. (Throughout the paper we set the speed of light and Planck's constant to unity.) The damping scale depends on the species being considered and its contribution to the energy density, and is the largest for neutrinos which are only coupled through weak interactions. In that case $N \sim\left(T / T_{d}^{\nu}\right)^{3}$ where $T_{d}^{\nu} \sim 1 \mathrm{MeV}$ is the temperature of neutrino decoupling. At the time of CDM decoupling $N \sim M / T_{d} \sim 10^{4}$ for the rest of the plasma, where $M$ is the mass of the CDM particle. In this paper we will consider modes of wavelength larger than $\lambda_{f}$, and so we neglect the effect of radiation diffusion damping and treat the plasma (without the CDM) as a perfect fluid.

The equations of motion for a perfect fluid during the radiation era can be solved analytically. We will use that solution here, following the notation of Ref. [12]. As usual we Fourier decompose fluctuations and study the behavior of each Fourier component separately. For a mode of comoving wavenumber $k$ in Newtonian gauge, the gravitational potential fluctuations are given by:

$$
\Phi=3 \Phi_{p}\left[\frac{\sin (\omega \eta)-\omega \eta \cos (\omega \eta)}{(\omega \eta)^{3}}\right]
$$

where $\omega=k / \sqrt{3}$ is the frequency of a mode and $\Phi_{p}$ is its primordial amplitude in the limit $\eta \rightarrow 0$. In this paper we use conformal time $\eta=\int d t / a(t)$ with $a(t) \propto t^{1 / 2}$ during the radiation-dominated era. The monopole $\Theta_{0}$ and dipole $\Theta_{1}$ of the photon distribution can be written in terms of the gravitational potential as:

$$
\begin{aligned}
& \Theta_{0}=\Phi\left(\frac{x^{2}}{6}+\frac{1}{2}\right)+\frac{x}{2} \Phi^{\prime} \\
& \Theta_{1}=-\frac{x^{2}}{6}\left(\Phi^{\prime}+\frac{1}{x} \Phi\right)
\end{aligned}
$$

where $x \equiv k \eta$ and a prime denotes a derivative with respect to $x$.

The solutions in equations (2) and (3) assume that both the sound speed and the number of relativistic degrees of freedom are constant over time. As a result of the QCD phase transition and of various particles becoming non-relativistic, both of these assumptions are not strictly correct. The vanishing sound speed during the QCD phase transition provides the most dramatic effect, but its imprint is on scales smaller than the ones we consider here because the transition occurs at a significantly higher temperature and only lasts for a fraction of the Hubble time [11].

Before the dark matter decouples kinematically we will treat it as a fluid which can exchange momentum with the plasma through particle collisions. At early times, the CDM fluid follows the motion of the plasma and is involved in its acoustic oscillations. The continuity and momentum equations for the CDM can be written as:

$$
\begin{aligned}
\dot{\delta}_{c}+\theta_{c} & =3 \dot{\Phi} \\
\dot{\theta}_{c}+\frac{\dot{a}}{a} \theta_{c} & =k^{2} c_{s}^{2} \delta_{c}-k^{2} \sigma_{c}-k^{2} \Phi+\tau_{c}^{-1}\left(\Theta_{1}-\theta_{c}\right)
\end{aligned}
$$

where a dot denotes an $\eta$-derivative, $\delta_{c}$ is the dark matter density perturbation, $\theta_{c}$ is the divergence of the dark matter velocity field and $\sigma_{c}$ denotes the anisotropic stress. In writing these equations we have followed Ref. [13]. The term $\tau_{c}^{-1}\left(\Theta_{1}-\theta_{c}\right)$ encodes the transfer of momentun between the radiation and CDM fluids and $\tau_{c}^{-1}$ provides the collisional rate of momentum transfer,

$$
\tau_{c}^{-1}=n \sigma \frac{T}{M} a,
$$

with $n$ being the number density of particles with which the dark matter is interacting, $\sigma(T)$ the average cross section for interaction and $M$ the mass of the dark matter 
particle. The relevant scattering partners are the standard model leptons which have thermal abundances. For detailed expressions of the cross section in the case of supersymmetric (SUSY) dark matter, see Refs. [10,7]. For our purpose, it is sufficient to specify the typical size of the cross section and its scaling with cosmic time,

$$
\sigma \approx \frac{T^{2}}{M_{\sigma}^{4}}
$$

where the coupling mass $M_{\sigma}$ is of the order of the weakinteraction scale $(\sim 100 \mathrm{GeV})$ for SUSY dark matter. This equation should be taken as the definition of $M_{\sigma}$, as it encodes all the uncertainties in the details of the particle physics model into a single parameter. The temperature dependance of the averaged cross section is a result of the available phase space. Our results are quite insensitive to the details other than through the decoupling time. Equating $\tau_{c}^{-1} / a$ to the Hubble expansion rate gives the temperature of kinematic decoupling:

$T_{d}=\left(\frac{M_{\sigma}^{4} M}{M_{p l}}\right)^{1 / 4} \approx 10 \mathrm{MeV}\left(\frac{M_{\sigma}}{100 \mathrm{GeV}}\right)\left(\frac{M}{100 \mathrm{GeV}}\right)^{1 / 4}$

The term $k^{2} c_{s}^{2} \delta_{c}$ in Eq. (4) results from the pressure gradient force and $c_{s}$ is the dark matter sound speed. In the tight coupling limit, $\tau_{c} \ll H^{-1}$ we find that $c_{s}^{2} \approx$ $f_{c} T / M$ and that the shear term is $k^{2} \sigma_{c} \approx f_{v} c_{s}^{2} \tau_{c} \theta_{c}$. Here $f_{v}$ and $f_{c}$ are constant factors of order unity. We will find that both these terms make a small difference on the scales of interest, so their precise value is unimportant.

By combining both equations in (4) into a single equation for $\delta_{c}$ we get

$$
\begin{aligned}
& \delta_{c}^{\prime \prime}+\frac{1}{x}\left[1+F_{\mathrm{v}}(x)\right] \delta_{c}^{\prime}+c_{s}^{2}(x) \delta_{c} \\
& =S(x)-3 F_{\mathrm{v}}(x) \Phi^{\prime}+\frac{x_{d}^{4}}{x^{5}}\left(3 \theta_{0}^{\prime}-\delta_{c}^{\prime}\right),
\end{aligned}
$$

where $x_{d}=k \eta_{d}$ and $\eta_{d}$ denotes the time of kinematic decoupling which can be expressed in terms of the decoupling temperature as,

$$
\begin{aligned}
\eta_{d}=2 t_{d}\left(1+z_{d}\right) \approx \frac{M_{p l}}{T_{0} T_{d}} & \approx 10 \mathrm{pc}\left(\frac{T_{d}}{10 \mathrm{MeV}}\right)^{-1} \\
& \propto M_{\sigma}^{-1} M^{-1 / 4}
\end{aligned}
$$

with $T_{0}=2.7 \mathrm{~K}$ being the present-day CMB temperature and $z_{d}$ being the redshift at kinematic decoupling. We have also introduced the source function,

$$
S(x) \equiv-3 \Phi^{\prime \prime}+\Phi-\frac{3}{x} \Phi^{\prime} .
$$

For $x \ll x_{d}$, the dark matter sound speed is given by

$$
c_{s}^{2}(x)=c_{s}^{2}\left(x_{d}\right) \frac{x_{d}}{x}
$$

where $c_{s}^{2}\left(x_{d}\right)$ is the dark matter sound speed at kinematic decoupling (in units of the speed of light),

$$
c_{s}\left(x_{d}\right) \approx 10^{-2} f_{c}^{1 / 2}\left(\frac{T_{d}}{10 \mathrm{MeV}}\right)^{1 / 2}\left(\frac{M}{100 \mathrm{GeV}}\right)^{-1 / 2}
$$

In writing (11) we have assumed that prior to decoupling the temperature of the dark matter follows that of the plasma. For the viscosity term we have,

$$
F_{v}(x)=f_{v} c_{s}^{2}\left(x_{d}\right) x_{d}^{2}\left(\frac{x_{d}}{x}\right)^{5}
$$

Free streaming after kinematic decoupling In the limit of the collision rate being much slower than the Hubble expansion, the CDM is decoupled and the evolution of its perturbations is obtained by solving a Boltzman equation:

$$
\frac{\partial f}{\partial \eta}+\frac{d x_{i}}{d \eta} \frac{\partial f}{\partial x_{i}}+\frac{d q_{i}}{d \eta} \frac{\partial f}{\partial q_{i}}=0
$$

where $f(\vec{x}, \vec{q}, \eta)$ is the distribution function which depends on time, position and comoving momentum $\vec{q}$. The comoving momentum 3-components are $d x_{i} / d \eta=q_{i} / a$. We use the Boltzman equation to find the evolution of modes that are well inside the horizon with $x \gg 1$. In the radiation era, the gravitational potential decays after horizon crossing (see Eq. 2). In this limit the comoving momentum remains constant, $d q_{i} / d \eta=0$ and the Boltzman equation becomes,

$$
\frac{\partial f}{\partial \eta}+\frac{q_{i}}{a} \frac{\partial f}{\partial x_{i}}=0
$$

We consider a single Fourier mode and write $f$ as,

$$
f(\vec{x}, \vec{q}, \eta)=f_{0}(q)\left[1+\delta_{F}(\vec{q}, \eta) e^{i \vec{k} \cdot \vec{x}}\right],
$$

where $f_{0}(q)$ is the unperturbed distribution,

$$
f_{0}(q)=n_{\mathrm{CDM}}\left(\frac{M}{2 \pi T_{\mathrm{CDM}}}\right)^{3 / 2} \exp \left[-\frac{1}{2} \frac{M q^{2}}{T_{\mathrm{CDM}}}\right]
$$

where $n_{\mathrm{CDM}}$ and $T_{\mathrm{CDM}}$ are the present-day density and temperature of the dark matter.

Our approach is to solve the Boltzman equation with initial conditions given by the fluid solution at a time $\eta_{*}$ (which will depend on $k$ ). The simplified Boltzman equation can be easily solved to give $\delta_{F}(\vec{q}, \eta)$ as a function of the initial conditions $\delta_{F}\left(\vec{q}, \eta_{*}\right)$,

$$
\delta_{F}(\vec{q}, \eta)=\delta_{F}\left(\vec{q}, \eta_{*}\right) \exp \left[-i \vec{q} \cdot \vec{k} \frac{\eta_{*}}{a\left(\eta_{*}\right)} \ln \left(\eta / \eta_{*}\right)\right] .
$$

The CDM overdensity $\delta_{c}$ can then be expressed in terms of the perturbation in the distribution function as, 


$$
\delta_{c}(\eta)=\frac{1}{n_{\mathrm{CDM}}} \int d^{3} q f_{0}(q) \delta_{F}(\vec{q}, \eta) .
$$

We can use (18) to obtain the evolution of $\delta_{c}$ in terms of its value at $\eta_{*}$,

$$
\delta_{c}(\eta)=\exp \left[-\frac{1}{2} \frac{k^{2}}{k_{f}^{2}} \ln ^{2}\left(\frac{\eta}{\eta_{*}}\right)\right]\left[\left.\delta\right|_{\eta_{*}}+\left.\frac{d \delta}{d \eta}\right|_{\eta_{*}} \eta_{*} \ln \left(\frac{\eta}{\eta_{*}}\right)\right],
$$

where $k_{f}^{-2}=\sqrt{\left(T_{d} / M\right)} \eta_{d}$. The exponential term is responsible for the damping of perturbations as a result of free streaming and the dispersion of the CDM particles after they decouple from the plasma. The above expression is only valid during the radiation era. The free streaming scale is simply given by $\int d t(v / a) \propto \int d t a^{-2}$ which grows logarithmically during the radiation era as in equation (20) but stops growing in the matter era when $a \propto t^{2 / 3}$.

Equation (20) can be used to show that even during the free streaming epoch, $\delta_{c}$ satisfies equation (8) but with a modified sound speed and viscous term. For $x \gg x_{d}$ one should use,

$$
\begin{aligned}
& c_{s}^{2}(x)=c_{s}^{2}\left(x_{d}\right)\left(\frac{x_{d}}{x}\right)^{2}\left[1+x_{d}^{2} c_{s}^{2}\left(x_{d}\right) \ln ^{2}\left(\frac{x}{x_{d}}\right)\right] \\
& F_{v}(x)=2 c_{s}^{2}\left(x_{d}\right) x_{d}^{2} \ln \left(\frac{x_{d}}{x}\right)
\end{aligned}
$$

The differences between the above scalings and those during the tight coupling regime are a result of the fact that the dark matter temperature stops following the plasma temperature but rather scales as $a^{-2}$ after thermal decoupling, which coincides with the kinematic decoupling. We ignore the effects of heat transfer during the fluid stage of the CDM because its temperature is controlled by the much larger heat reservoir of the radiation-dominated plasma at that stage.

To obtain the transfer function we solve the dark matter fluid equation until decoupling and then evolve the overdensity using equation (20) up to the time of matterradiation equality. In practice, we use the fluid equations up to $x_{*}=10 \max \left(x_{d}, 10\right)$ so as to switch into the free streaming solution well after the gravitational potential has decayed. In the fluid equations, we smoothly match the sound speed and viscosity terms at $x=x_{d}$. As mentioned earlier, because $c_{s}\left(x_{d}\right)$ is so small and we are interested in modes that are comparable to the size of the horizon at decoupling, i.e. $x_{d} \sim$ few, both the dark matter sound speed and the associated viscosity play only a minor role, and our simplified treatment is adequate.

In Figure 1 we illustrate the time evolution of modes during decoupling for a variety of $k$ values. The situation is clear. Modes that enter the horizon before kinematic decoupling oscillate with the radiation fluid. This behavior has two important effects. In the absence of the

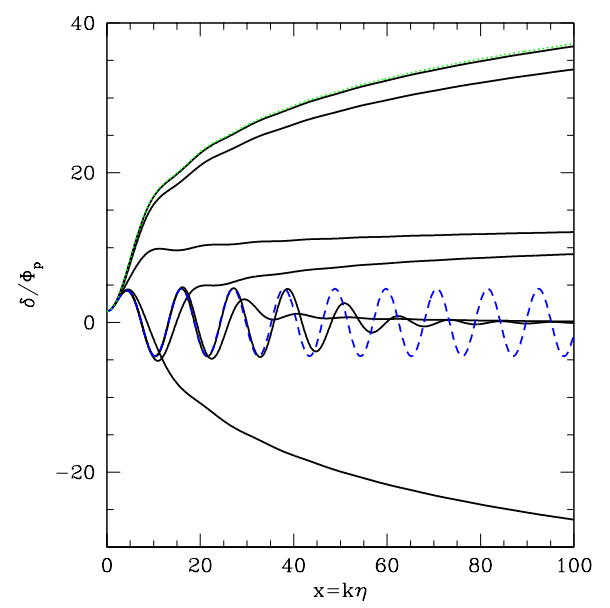

FIG. 1. The normalized amplitude of CDM fluctuations $\delta / \Phi_{P}$ for a variety of modes with comoving wavenumbers $\log \left(k \eta_{d}\right)=(0,1 / 3,2 / 3,1,4 / 3,5 / 3,2)$ as a function of $x \equiv k \eta$, where $\eta=\int_{0}^{t} d t / a(t)$ is the conformal time coordinate. The dashed line shows the temperature monopole $3 \theta_{0}$ and the dotted curve shows the evolution of a mode that is uncoupled to the cosmic plasma.

coupling, modes receive a "kick" by the source term $S(x)$ as they cross the horizon. After that they grow logarithmically. In our case, modes that entered the horizon before kinematic decoupling follow the plasma oscillations and thus miss out on both the horizon "kick" and the beginning of the logarithmic growth. Second, the decoupling from the radiation fluid is not instantaneous and this acts to further damp the amplitude of modes with $x_{d} \gg 1$. This effect can be understood as follows. Once the oscillation frequency of the mode becomes high compared to the scattering rate, the coupling to the plasma effectively damps the mode. In that limit one can replace the forcing term $\Theta_{0}^{\prime}$ by its average value, which is close to zero. Thus in this regime, the scattering is forcing the amplitude of the dark matter oscillations to zero. After kinematic decoupling the modes again grow logarithmically but from a very reduced amplitude. The coupling with the plasma induces both oscillations and damping of modes that entered the horizon before kinematic decoupling. This damping is different from the free streaming damping that occurs after kinematic decoupling.

In Figure 2 we show the resulting transfer function of the CDM overdensity. The transfer function is defined as the ratio between the CDM density perturbation amplitude $\delta_{c}$ when the effect of the coupling to the plasma is included and the same quantity in a model where the CDM is a perfect fluid down to arbitrarily small scales (thus, the power spectrum is obtained by multiplying the standard result by the square of the transfer function). This function shows both the oscillations and the damping signature mentioned above. The peaks occur at multipoles of the horizon scale at decoupling, 


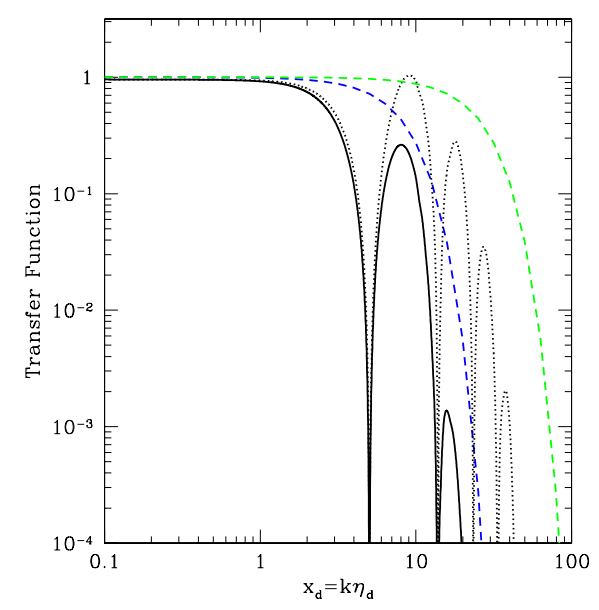

FIG. 2. Transfer function of the CDM density perturbation amplitude (normalized by the primordial amplitude from inflation). We show two cases: (i) $T_{d} / M=10^{-4}$ and $T_{d} / T_{\text {eq }}=10^{7}$; (ii) $T_{d} / M=10^{-5}$ and $T_{d} / T_{\text {eq }}=10^{7}$. In each case the oscillatory curve is our result and the other curve is the free-streaming only result that was derived previously in the literature $[4,7,8]$.

$$
k_{\text {peak }}=(8,15.7,24.7, . .) \eta_{d}^{-1} \propto \frac{M_{p l}}{T_{0} T_{d}} .
$$

This same scale determines the "oscillation" damping. The free streaming damping scale is,

$$
\eta_{d} c_{d}\left(\eta_{d}\right) \ln \left(\eta_{e q} / \eta_{d}\right) \propto \frac{M_{p l} M^{1 / 2}}{T_{0} T_{d}^{3 / 2}} \ln \left(T_{d} / T_{\mathrm{eq}}\right),
$$

where $T_{\text {eq }}$ is the temperature at matter radiation equality, $T_{\text {eq }} \approx 1 \mathrm{eV}$. The free streaming scale is parametrically different from the "oscillation" damping scale. However for our fiducial choice of parameters for the CDM particle they roughly coincide.

The vanishing of the sound speed during the QCD phase transition amplifies perturbations which have $\omega \Delta \eta_{Q C D}>1$, where $\Delta \eta_{Q C D}$ is the duration of the transition. In Figure 2 the affected modes are those with $x_{d}=k \eta_{d}>\sqrt{3}\left(\eta_{Q C D} / \Delta \eta_{Q C D}\right)\left(\eta_{d} / \eta_{Q C D}\right)$. Typical values of $\eta_{d} / \eta_{Q C D} \sim 10-15$ and $\eta_{Q C D} / \Delta \eta_{Q C D} \sim 3-10$ relate this condition to modes with $x_{d}>50-260$. Thus the affected scales are severely damped by the effects considered in this paper.

Finally we want to stress the fact that the damping scale is significantly smaller than the scales observed directly in the Cosmic Microwave Background or through large scale structure surveys. For example, the ratio of the damping scale to the scale that entered the horizon at equality is $\eta_{d} / \eta_{e q} \sim T_{e q} / T_{d} \sim 10^{-7}$ and to our present horizon $\eta_{d} / \eta_{0} \sim\left(T_{e q} T_{0}\right)^{1 / 2} / T_{d} \sim 10^{-9}$. In the context of inflation, these scales were created 16 and $20 e$-folds apart. Given the large extrapolation, one could certainly imagine that a change in the spectrum could alter the shape of the power spectrum around the damping scale.
However, for smooth inflaton potentials with small departures from scale invariance this is not likely to be the case. On scales much smaller than the horizon at matter radiation equality, the spectrum of perturbations density before the effects of the damping are included is approximately,

$$
\begin{aligned}
\Delta^{2}(k) \propto & \exp \left[(n-1) \ln \left(k \eta_{e q}\right)+\frac{1}{2} \alpha^{2} \ln \left(k \eta_{e q}\right)^{2}+\cdots\right] \\
& \times \ln ^{2}\left(k \eta_{e q} / 8\right)
\end{aligned}
$$

where the first term encodes the shape of the primordial spectrum and the second the transfer function. Primordial departures from scale invariance are encoded in the slope $n$ and its running $\alpha$. The effective slope at scale $k$ is then,

$$
\frac{\partial \ln \Delta^{2}}{\partial \ln k}=(n-1)+\alpha \ln \left(k \eta_{e q}\right)+\frac{2}{\ln \left(k \eta_{e q} / 8\right)} .
$$

For typical values of $(n-1) \sim 1 / 60$ and $\alpha \sim 1 / 60^{2}$ the slope is still positive at $k \sim \eta_{d}^{-1}$, so the cut-off in the power will come from the effects we calculate rather than from the shape of the primordial spectrum. However given the large extrapolation in scale, one should keep in mind the possibility of significant effects resulting from the mechanisms that generates the density perturbations.

Implications We have found that acoustic oscillations, a relic from the epoch when the dark matter coupled to the cosmic radiation fluid, truncate the CDM power spectrum on a comoving scale larger than effects considered before, such as free-streaming and viscosity $[4,7,8]$. For SUSY dark matter, the minimum mass of dark matter clumps that form in the universe is therefore increased by more than an order of magnitude to a value of *

$$
\begin{aligned}
M_{\text {cut }} & =\frac{4 \pi}{3}\left(\frac{\pi}{k_{\text {cut }}}\right)^{3} \Omega_{M} \rho_{\text {crit }} \\
& \simeq 10^{-4}\left(\frac{T_{d}}{10 \mathrm{MeV}}\right)^{-3} M_{\odot},
\end{aligned}
$$

where $\rho_{\text {crit }}=\left(H_{0}^{2} / 8 \pi G\right)=9 \times 10^{-30} \mathrm{~g} \mathrm{~cm}^{-3}$ is the critical density today, and $\Omega_{M}$ is the matter density for the concordance cosmological model [2]. We define the cutoff wavenumber $k_{\text {cut }}$ as the point where the transfer function first drops to a fraction $1 / e$ of its value at $k \rightarrow 0$. This corresponds to $k_{\text {cut }} \approx 3.3 \eta_{d}^{-1}$.

Recent numerical simulations $[15,16]$ of the earliest and smallest objects to have formed in the universe [17], need

\footnotetext{
${ }^{*}$ Our definition of the cut-off mass follows the convention of the Jeans mass, which is defined as the mass enclosed within a sphere of radius $\lambda_{\mathrm{J}} / 2$ where $\lambda_{\mathrm{J}} \equiv 2 \pi / k_{\mathrm{J}}$ is the Jeans wavelength [14].
} 
to be redone for the modified power spectrum that we calculated in this paper. Although it is difficult to forecast the effects of the acoustic oscillations through the standard Press-Schechter formalism [18], it likely that the results of such simulations will be qualitatively the same as before except that the smallest clumps would have a mass larger than before (as given by Eq. 26).

Potentially, there are several observational signatures of the smallest CDM clumps. As pointed out in the literature $[15,19]$, the smallest CDM clumps could produce $\gamma$ rays through dark-matter annihilation in their inner density cusps, with a flux in excess of that from nearby dwarf galaxies. If a substantial fraction of the Milky Way halo is composed of CDM clumps with a mass $\sim 10^{-4} M_{\odot}$, the nearest clump is expected to be at a distance of $\sim 4 \times 10^{17}$ $\mathrm{cm}$. Given that the characteristic speed of such clumps is a few hundred $\mathrm{km} \mathrm{s}^{-1}$, the $\gamma$-ray flux would therefore show temporal variations on the relatively long timescale of a thousand years. Passage of clumps through the solar system should also induce fluctuations in the detection rate of CDM particles in direct search experiments.

Other observational effects have rather limited prospects for detectability. Because of their relatively low-mass and large size $\left(\sim 10^{17} \mathrm{~cm}\right)$, the CDM clumps are too diffuse to produce any gravitational lensing signatures (including femto-lensing [20]), even at cosmological distances. They could, however, have an effect on the time delay of the signal from transient cosmological sources. We illustrate this effect by considering a characteristic CDM clump with a size $\sim 10^{17} \mathrm{~cm}$ and a mass of $10^{-4} M_{\odot}$ (corresponding to formation redshift $\sim 50$ ). If a major fraction of the intergalactic CDM is in the form of these clumps (amounting to a mean mass density of $\sim 3 \times 10^{-30} \mathrm{~g} \mathrm{~cm}^{-3}$ ), then the mean separation between clumps is $\sim 6 \times 10^{19} \mathrm{~cm}=20 \mathrm{pc}$. The gravitational potential would then fluctuate on this length scale everywhere in the universe with an amplitude of $\left(\Phi / c^{2}\right) \sim 2 \times 10^{-19}$. Now, consider a compact source at cosmological distances with a size comparable to the distance between clumps, $\sim 10$ pc. Photons emerging from different regions of the source will encounter different gravitational potential delays (the so-called Shapiro time delay [21]) along their path due to the fact that they traverse CDM clumps at different impact parameters. For a source that is much larger than $\sim 20 \mathrm{pc}$, the effect of multiple CDM clumps would average out because the source would sample the full distribution of impact parameters with small statistical fluctuations around the mean time delay. The mean Shapiro delay from the network of CDM clumps along the line-of-sight to a cosmological source is $\sim\left(\Phi / c^{2}\right) t_{H} \sim 0.1$ sec, where $t_{H}$ is the light travel time across the observable universe. The total number of clumps along the line-ofsight is $N \sim(4 \mathrm{Gpc} / 20 \mathrm{pc}) \sim 2 \times 10^{8}$. This implies that the variance in time delays across a source of size $\sim 10 \mathrm{pc}$ would be suppressed by a factor of $\sim 1 / \sqrt{N} \sim 10^{-4}$ relative to the mean time delay, i.e. it would have a mag- nitude $\sim 10 \mu$ sec. $\gamma$-ray bursts may possess variability on such a timescale (which would have been smoothed out if their source had a size $\sim 10 \mathrm{pc}$ ), but their estimated source size is $R<0.1 \mathrm{pc}$ [22] and so the temporal smoothing effect for them is smaller by another factor $>(20 / 0.1)=2 \times 10^{2}$, which leads to a variance in the delay timescale of less than a microsecond. Unfortunately this time delay appears unmeasurable, since the variability of any source of apparent dimension $R$ would be expected to be smoothed-out on a timescale, $\sim R / \gamma c$, where $\gamma$ is the Lorentz factor of the emitting material (which is $<10^{3}$ for $\gamma$-ray bursts).

The smallest CDM clumps should not affect the intergalactic baryons which have a much larger Jeans mass [17]. However, once objects above $\sim 10^{6} M_{\odot}$ start to collapse at redshifts $z<30$, the baryons would be able to cool inside of them via molecular hydrogen transitions and the interior baryonic Jeans mass would drop. The existence of dark matter clumps could then seed the formation of the first stars inside these objects [23].

Finally, we note that the smallest CDM clumps may have a dynamical effect on wide orbits of test particles near the outer edge the solar system after the effects of the known planets have been modeled to a high precision [24].

Acknowledgments. This work was supported in part by NASA grant NAG 5-13292, NSF grants AST-0071019, AST-0204514 (for A.L.) and by NSF grants AST0098606, PHY-0116590 and the David \& Lucille Packard Foundation Fellowship (for M.Z.).

[1] G. Jungman, M. Kamionkowski, \& K. Griest,Phys. Rep. 267, 195 (1996); L. Bergstrom, Rep. Prog. Phys. 63, 793 (2000); R. J. Gaitskell, Ann. Rev. of Nuclear and Particle Science 54, 315 (2004).

[2] D. N. Spergel, et al., Astrophys. J. Suppl. 148, 175 (2003).

[3] M. Milgrom, New Astron. Rev. 46, 741 (2002); J. D. Bekenstein, Phys.Rev. D 70, 083509 (2004).

[4] A. M. Green, S. Hofmann, \& D. J. Schwarz, Mon. Not. R. Astr. Soc., 353, L23 (2004).

[5] M. Battaglia, A. De Roeck, J. R. Ellis, F. Gianotti, K. A. Olive and L. Pape, Eur. Phys. J. C 33, 273 (2004) [arXiv:hep-ph/0306219].

[6] E. A. Baltz, arXiv:astro-ph/0412170.

[7] A. M. Green, S. Hofmann, \& D. J. Schwarz, preprint astro-ph/0503387 (2005).

[8] S. Hofmann, D. J Schwarz, \& H. Stöker, Phys. Rev. D 64, 083507 (2001).

[9] D. J. Eisenstein, et al. Astrophys. J., submitted, preprint astro-ph/0501171 (2005).

[10] X. Chen, M., Kamionkowski, \& X. Zhang, Phys. Rev. D 64, 021302 (2001). 
[11] C. Schmid, D. J. Schwarz, \& P. Widerin, Phys. Rev. D, 59, 043517 (1999).

[12] S. Dodelson, Modern Cosmology, (Acacdemic Press: San Diego, CA), (2003).

[13] C., Ma, \& E. Bertschinger, Astrophys. J. 455, 7 (1995).

[14] Z. Haiman, A. Thoul, \& A. Loeb, Astrophys. J. 464, 523 (1996).

[15] J. Diemand, B. Moore, \& J. Stadel, Nature 433, 389 (2005).

[16] L. Gao, S. D. M. White, A. Jenkins, F. Stoehr, \& V. Springel, Mon. Not. R. Astron. Soc. 355, 819 (2004).

[17] R. Barkana, \& A. Loeb, Phys. Rep. 349, 125 (2001).

[18] W. H. Press, \& P. Schechter, Astrophys. J. 193, 437 (1974).

[19] F. Stoehr, S. D. M. White, V. Springel, G. Tormen, \& N. Yoshida, Mon. Not. R. Astron. Soc. 345, 1313 (2003).

[20] A. Gould, Astrophys. J. Lett. 386, 5 (1992); K. Z. Stanek, B. Paczynski \& J. Goodman, Astrophys. J. Lett. 413, 7 (1993); A. Ulmer \& J. Goodman Astrophys. J. 442, 67 (1995).

[21] I. I. Shapiro, Phys. Rev. Lett. 13, 789 (1964).

[22] T. Piran, Phys. Rep., 333, 529 (2000).

[23] V. Bromm, \& R. B. Larson, Ann. Rev. Astr. \& Astrophys. 42, 79 (2004).

[24] A. Loeb \& C. Steinhardt, in preparation (2005). 